\title{
cRGDfK-Grafted Small-Size Quercetin Micelles For Enhancing Therapy Efficacy Of Active Ingredient From The Chinese Medicinal Herb
}

This article was published in the following Dove Press journal: International Journal of Nanomedicine

\author{
Pengcheng $\mathrm{Xu}$ (I) $^{1}$ \\ Haisheng Wang ${ }^{2}$ \\ Hongxiang $\mathrm{Hu}^{3}$ \\ Yong $\mathrm{Ye}^{4}$ \\ Yu Dong' \\ Suxin $\mathrm{Li}^{3}$ \\ Dong Mei $\mathbb{D D}^{3}$ \\ Zhaoming Guo $\mathbb{D}^{3}$ \\ Dan Wang ${ }^{3}$ \\ Yanxue Sun' \\ Tengfei Yu' \\ Junchan Qiao' \\ Qiang Zhang ${ }^{3}$ \\ 'Department of Pharmaceutical \\ Engineering, College of Pharmacy, Inner \\ Mongolia Medical University, Hohhot \\ 0I0I 10, People's Republic of China; \\ ${ }^{2}$ Department of Biochemistry, College of \\ Basic Medical Sciences, Inner Mongolia \\ Medical University, Hohhot 0I0II0, \\ People's Republic of China; ${ }^{3}$ Beijing Key \\ Laboratory of Molecular Pharmaceutics \\ and New Drug Delivery Systems, \\ Department of Pharmaceutics, School of \\ Pharmaceutical Sciences, Peking \\ University, Beijing 100191, People's \\ Republic of China; ${ }^{4}$ Department of \\ Pharmaceutics, College of Pharmacy, \\ Guangxi Medical University, Nanning \\ 53002I, People's Republic of China
}

Correspondence: Qiang Zhang Tel +86-010-8280279l

Email zqdodo@bjmu.edu.cn
Background: As an active ingredient of Chinese herbal medicine, quercetin (QU) can significantly induce apoptosis of tumor cells and give play to other effect such as decreasing both fibroblast population and collagen in cancer cell nest. However, the antitumor efficacy of quercetin was mostly evaluated at cellular level and rarely developed in vivo by intravenous injection, which may be ascribed to its inferior physicochemical properties including water insolubility, short plasma half-time, and insufficient enrichment in the tumor tissues.

Methods: The DSPE-PEG was used to construct quercetin-loaded micelles, and the integrin ligand cRGDfK was grafted to modify the nanocarrier for enhancing its cancer-specific homing. The MALDI-TOF-MS, DLS, TEM, and UV were orderly operated to characterize guidance molecules and micelles by morphology, size distribution, Zeta potential, and drug encapsulation efficiency. In addition, the surface plasmon resonance study and real-time confocal analysis were employed to demonstrate $\alpha_{\mathrm{v}} \beta_{3}$ integrin-overexpressing B16 cellsspecific binding and uptake. After further pharmacodynamics studies in vitro and in vivo, we also evaluate systemic toxicity about cRGDfK-PM-QU.

Results: The cRGDfK was successfully stitched with DSPE-PEG and modified on the surface of micelles. The ligand modification enhanced the negative charges of the micelles, but it did not induce significant changes in particle size. The quercetin micelles were about $15 \mathrm{~nm}$ in size and negatively charged, and had spherical morphology and high drug encapsulation efficiency. In vitro, the cRGDfK-modified micelles (cRGDfK-PM) showed $\alpha_{\mathrm{v}} \beta_{3}$ integrin-overexpressing B16 cells-specific binding and uptake, and cRGDfK-PM-QU (QU loaded in cRGDfK-PM) induced more significant cell apoptosis and cytotoxic effects against B16 tumor cells than counterpart micelles (PM-QU). In vivo, the cRDGfK modification enhanced enrichment in B16 tumor tissue, improved the therapeutic efficacy of the quercetin-loaded micelles against B16 tumor, and exhibited lower systemic and pulmonary toxicity compared with counterpart micelles in the mouse mode.

Conclusion: Quercetin as a natural product has triggered increasing interest in the antitumor field. In this study, cRGDfK-modified DSPE-PEG micelles significantly optimized quercetin therapeutic efficacy and pulmonary toxicity as well as lowered systemic toxicity.

Keywords: quercetin, micelles, cRGDfK, integrin

\section{Introduction}

Quercetin (3, 3', 4', 5, 7-penthydroxy flavone), an active ingredient of Chinese herbal medicine, can typically inhibit tumor cells growth. ${ }^{1}$ In vitro studies have shown that quercetin can remarkably induce cell apoptosis, restrain angiogenesis, decrease both fibroblast population and collagen, and finally, resist several proliferating human 
carcinoma cells, including leukemia, ovarian cancer, breast adenocarcinoma, colon carcinoma, and prostate adenocarcinoma. ${ }^{1-11}$

The characteristics of quercetin which include water insolubility, poor bioavailability, poor permeability, and short half-time in vivo have hindered its clinical application. ${ }^{12}$ As far as we know, the nano-drug delivery system can be employed to solve the above problems. ${ }^{13-15}$ Although quercetin nanoparticles are widely constructed, their antitumor effects in animal models are rarely reported. Recently, oral administration of quercetin nanoparticles was reported. ${ }^{16}$ Despite the convenience of oral administration, the administration route faces the problem of first-pass effect resulting in low bioavailability. In addition, generally, the oral formulations do not show tumor tissue-specific distribution. In contrast with oral administration, parenteral administration, which can bypass bioavailability barriers of oral ingestion, is often used in clinical cancer treatment. ${ }^{17-19}$

A great deal of block polymer can self-assemble into nanocarrier to deliver therapeutic agents. DSPE-PEG, a widely used phospholipids-polymer approved by the Food and Drug Administration (FDA) for medical applications, ${ }^{20}$ shows satisfying biocompatibility and biodegradability. ${ }^{21}$ Generally, the micelles prepared by DSPE-PEG exhibit high transport capacity, favorable release profile, ${ }^{22}$ and excellent stability in medium incorporating human serum. ${ }^{23}$ Nanocarriers in use of DSPEPEG can also achieve prolonged blood circulation time, improved drug stability, and enhanced drug encapsulation yield while significant toxicity is not found. ${ }^{24-26}$ Given the advantages of DSPE-PEG earlier, the amphiphilic polymer was used in this study to encapsulate quercetin.

cRGDfK, a cyclic derivative of RGD peptide, shows improved chemical stability compared to the linear RGD peptides. ${ }^{27}$ On the other hand, owing to structural rigidity which constrains the peptides trapped in the active conformation, previous reports have indicated that cyclic peptides improve tumor targeting efficacy. ${ }^{28,29}$ cRGDfK demonstrates high affinity and binding specificity for $\alpha_{\mathrm{v}}$ $\beta_{3}$ integrin. ${ }^{30-32}$ These studies have proved that the cyclic peptides show higher $\alpha_{v} \beta_{3}$ integrin-binding affinity than linear ones. ${ }^{33,34}$ Given its superiority, cRGDfK is assigned as grafted peptide to enhance the uptake of quercetin micelles by the $\alpha_{v} \beta_{3}$ integrin receptor overexpressed melanoma cells and the distribution in tumor tissue.

Based on the earlier descriptions and discussions, DSPEPEG micelles modified by cRGDfK were prepared to encapsulate and deliver quercetin. Surface plasmon resonance (SPR) technique was used to investigate the interactions between cRGDfK-PM-QU and the $\alpha_{v} \beta_{3}$-integrin receptor overexpressed melanoma B16 cells. The cell uptake of the nanocarrier was measured by confocal microscope and flow cytometry, and then the cytotoxicity and cell apoptosis assays were also carried out. Furthermore, the near-infrared fluorescence imaging was employed to evaluate the influence of cRGDfK modification on in vivo distribution and the therapeutic effects were assessed in tumor-bearing nu/nu mice.

\section{Materials And Methods Materials}

Quercetin was purchased from Sinopharm Chemical Reagent Co., Ltd (Shanghai, China). NHS- PEG2000-DSPE (Mw 2986) and DSPE-PEG2000 (Mw 2922) were purchased from NOF Corporation (Tokyo, Japan). The Cyclic-RGDfK (cRGDfK) peptide (Mw 604) was synthesized by China Peptides Co., Ltd (Shanghai, China). Near-infrared fluorescence dye DiR was obtained from Biotium, Inc. (Hayward, USA). Sulforhodamine B and Tris base were obtained from Sigma-Aldrich (St. Louis, MO, USA). Annexin V-FITC/PI kit was obtained from Beyotime Biotech Co., Ltd (Shanghai, China).

B16 cells were obtained from the Institute of Basic Medical Science, Chinese Academy of Medical Science (Beijing, China). B16 cells were cultured in RPMI Medium-1640 (Macgeme Biotech Co., Ltd, Beijing, China) added with $10 \%$ fetal bovine serum (FBS) and two antibiotics $(100 \mathrm{mg} / \mathrm{mL}$ streptomycin, $100 \mathrm{U} / \mathrm{mL}$ penicillin) at $37^{\circ} \mathrm{C}$ in $5 \% \mathrm{CO}_{2}$ atmosphere.

Male $\mathrm{nu} / \mathrm{nu}$ mice of $18-20 \mathrm{~g}$ were selected and purchased from Peking University Health. All animal studies were reviewed according to the Guide for Care and Use of the Laboratory Animal and approved by the Ethics Committee of Peking University Health Science Center.

\section{Synthesis Of cRGDfK Peptides Copolymer}

cRGDfK peptides were prepared according to the previously reported method. ${ }^{35}$ The reaction lasted for $24 \mathrm{hrs}$ and was monitored by thin-layer chromatography (TLC). The final reaction mixture was poured into a dialysis bag (MW cut off, $3500 \mathrm{Da}$ ) to dialyze against distilled water for $48 \mathrm{hrs}$. Eventually, the product in the dialysis bag was lyophilized and characterized by MALD-TOF-MS. 


\section{Preparation Of The Drug-Loaded Polymeric Micelles}

PEG-DSPE was dissolved in acetonitrile, and quercetin was dissolved in the mixture of ethanol and methanol (1:2). The two organic solutions were combined in a pearshaped flask, with different weight proportions of the drug and PEG-DSPE. The solvents were then separated by rotary vacuum evaporation at $30^{\circ} \mathrm{C}$ until a dry film formed. The prepared film was quickly hydrated in PBS (pH 7.4) solution at $30^{\circ} \mathrm{C}$ to get the micelle solution. After centrifugating the solution at $10,000 \mathrm{rpm}$ for 3 mins to remove the free quercetin, the final micelles were obtained. The other micelles including carrying Dir (PM-Dir, cRGDfkPM-Dir) were prepared by the same method.

\section{Characterization Of The Drug-Loaded Micelles}

The particle size and zeta-potential of the micelles were measured using a Malvern Zetasizer Nano ZS (Malvern, United Kingdom). Nanoparticle morphology was featured using transmission electron microscope (JEOL, JEM-200CX, Japan). The encapsulation efficiency (EE) of quercetin (QU) in the micelles was analyzed by UV. X-ray diffractometer (Dmax 2400, Rigaku Corporation, Japan) was used to characterize the free QU powder, the lyophilized blank micelles, the mixture of the free QU powder and the lyophilized blank micelles, the lyophilized active drug-loaded micelles, and the lyophilized passive drug-loaded micelles.

\section{SPR Analysis}

Simply, the CM5 chip was pretreated with 1-ethyl3-[3-dimethylaminopropyl] carbodiimide hydrochloride (EDC) and N-hydroxysuccinimide (NHS) at the beginning of the experiment. Next, B16 cell suspension $\left(2 \times 10^{5} \mathrm{~mL}^{-1}\right)$ was injected to link the cells covalently with the sensor chip until the response (RU) signal ran up to about 300 . Subsequently, the sensor chip was blocked with acetamide. After the response signal was stable, the micelle solution was injected at the flow rate of $2.5 \mu \mathrm{L} / \mathrm{min}$.

\section{Cell Uptake Of The Micelles By BI6 Cells}

B16 cells were seeded on a glass-bottom dish and cultured for $24 \mathrm{hrs}$. Afterwards, PM-QU or cRDGfK-PM-QU was added to make the final drug concentration of $10 \mu \mathrm{g} / \mathrm{mL}$. After incubation at $37^{\circ} \mathrm{C}$ for different periods, the medium was discarded and the cells were washed by PBS, and then imaged by confocal microscope (Leica TCS SP8, Germany).

\section{Cell Uptake By Flow Cytometry}

B16 cells were seeded in 12-well plates. The initial density of cells was approximately $5 \times 10^{5}$ cells per well. PM-QU or cRGDfK-PM-QU was added to the medium to make the final drug concentration of $20 \mu \mathrm{g} / \mathrm{mL}$ with incubation for 2 hrs, and then the cells were gathered by trypsin treatment and centrifugation. After washing the cells by PBS twice, the fluorescence intensity of the cells was measured by FACScan flow cytometer (FACScan, Becton Dickinson, San Jose, CA).

\section{In Vitro Cytotoxicity Assay}

B16 cells were seeded in 96-well plate with about 5000 cells per well. After being cultured for $24 \mathrm{hrs,} \mathrm{the} \mathrm{medium} \mathrm{was}$ replaced by fresh medium containing PM-QU or cRGDfKPM-QU, with serial concentrations of QU. Here, to follow with incubation at $37^{\circ} \mathrm{C}$ for $48 \mathrm{hrs}$, SRB method was used to analyze the viability of the cells. The absorbance was measured using Multiskan FC microplate reader (Thermo Scientific, USA), the wavelength was $540 \mathrm{~nm}$.

\section{Cell Apoptosis Analysis}

B16 cells were seeded in 12-well plates at a density of $5 \times$ $10^{5}$ cells per well and cultured overnight. Afterwards, the cells were treated with PM-QU or cRGDfK-PM-QU and the final concentration of QU in each well was $5 \mu \mathrm{g} / \mathrm{mL}$. The untreated cells were used as negative control. After incubation for $24 \mathrm{hrs}$, the cells were gathered. The Annexin V/PI apoptosis test was carried out according to the protocol and, analyzed by FACScan flow cytometry.

\section{Distribution Of The Micelles In}

\section{Tumor-Bearing Mice}

Approximately $6 \times 10^{6}$ of B16 cells were subcutaneously injected into the right flank of the mice. When the tumor volume reached about $500 \mathrm{~mm}^{3}$, the mice were divided randomly into two groups (3 mice each group). PM-Dir or cRGDfK-PM-Dir was injected into the mice via the tail vein ( $2 \mu \mathrm{g} \mathrm{Dir} /$ mouse). At different time intervals of 1,3 , 7, 13, 26, and 48-hr post-injection, the near-infrared fluorescence images of the mice were obtained (Carestream, Fx Pro, USA). Afterwards, the mice were sacrificed and the tumor tissues and major organs were collected and imaged.

\section{Antitumor Effects And Systemic Toxicity Of The Micelles}

Approximately $6 \times 10^{6}$ of B16 cells were subcutaneously injected into the right flank of the mice to establish melanoma 
A

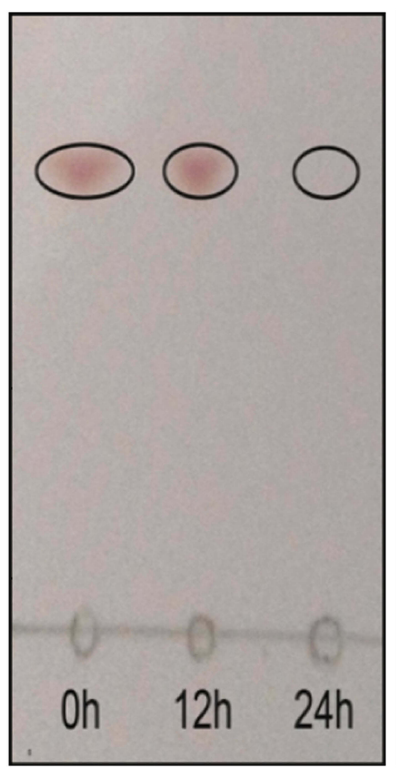

B

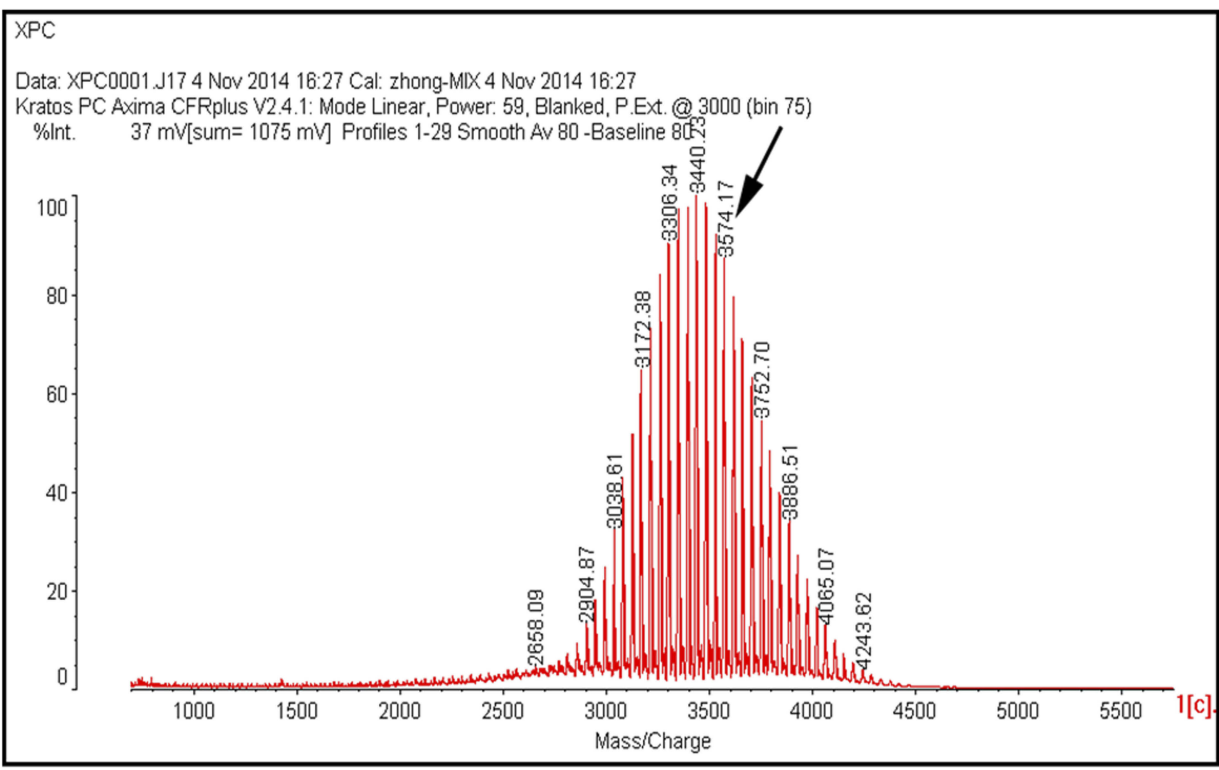

Figure I Copolymer synthesis. (A) TLC for the reaction endpoint determination. (B) MALDI-TOF-MS spectra for confirmations of reaction product of cRGDfK-PEG-DSPE.

mouse model. At the time, the tumor grew to $50-100 \mathrm{~mm}^{3}$ on the 8 th day, the mice were divided randomly into 3 groups with 3 mice in each group. The mice were consecutively treated by PM-QU or cRGDfK-PM-QU at a QU dose of $50 \mathrm{mg} / \mathrm{kg}$ body weight for 10 days, the mice treated by PBS were used as control. The body weights were recorded every other day. At the end of drug administration, further observations were carried out for another 5 days. Finally, the mice were sacrificed and, the tumor tissues and the main organs of heart, liver, spleen, kidney, and lung were collected. The anti-tumor effects were evaluated by tumor weight difference. The main organs were sliced and H\&E stained to evaluate the systemic toxicity.

\section{Statistics Analysis}

All experiments were performed repeatedly at least three times and quantitative data are expressed in the form of mean \pm standard deviation (SD) unless otherwise stated in this study. The results were evaluated by Student's $t$-test or one-way analysis of variance. $\mathrm{P}$ values less than 0.05 or less than 0.01 were considered as significant or highly significant.

\section{Results And Discussion \\ Characterization Of cRGDfK-Conjugated PEG-DSPE}

cRGDfK peptide as a ligand was successfully conjugated to DSPE-PEG-NHS by a nucleophilic substitution reaction. The reaction was monitored by TLC (Figure 1A). The final product molecular weight (M. W.) was confirmed by MALDI-TOF-MS (Figure 1B) and was accordant with the theoretical M. W. of cRGDfK-PEG-DSPE (3575.2). Synthesis scheme for cRGDfK-PEG-DSPE copolymer is shown in Figure S1

\section{Characterizations Of Quercetin Micelles}

Table 1 depicts the physicochemical properties of different micelles. The mean value of particle size of each PM was

Table I Characteristics Of Various Drug-Loaded DSPE-PEG Micelles (Mean $\pm s d, n=3$ )

\begin{tabular}{|l|l|l|l|l|}
\hline Formulations & Size(nm) & PDI & Zeta Potential $(\mathbf{m V})$ & EE $^{\mathbf{a}}(\%)$ \\
\hline Blank-PM & $12.19 \pm 0.37$ & $0.107 \pm 0.032$ & $-3.28 \pm 0.77$ & - \\
Blank-cRGDfK-PM & $13.22 \pm 1.06$ & $0.134 \pm 0.035$ & $-17.13 \pm 3.52$ & - \\
PM-QU & $13.91 \pm 2.14$ & $0.169 \pm 0.062$ & $-10.31 \pm 4.81$ & $88.4 \pm 2.16$ \\
cRGDfK-PM-QU & $15.34 \pm 2.73$ & $0.182 \pm 0.084$ & $-29.86 \pm 5.62$ & $86.7 \pm 2.73$ \\
\hline
\end{tabular}

Note: ${ }^{a}$ Encapsulation efficiency. 
within the range of $15-25 \mathrm{~nm}$, with a small polydispersity index (PDI) less than 0.2. As a result of cRGDfK modification, particle sizes were slightly enlarged in comparison with unmodified micelles. TEM images confirmed that cRGDfKPM-QU had a spherical morphology with a diameter of about $15 \mathrm{~nm}$, which was basically consistent with that measured by dynamic light scattering (Figure 2C and D). In the study, all the micelle systems were negatively charged. Specifically, the zeta potential of cRGDfK-PM-QU was about $-30 \mathrm{mV}$, much higher than that of PM-QU (Figure 2E and F). It means that cRGDfK modification greatly enhanced the surface charges of the micelles. Recent researches have indicated
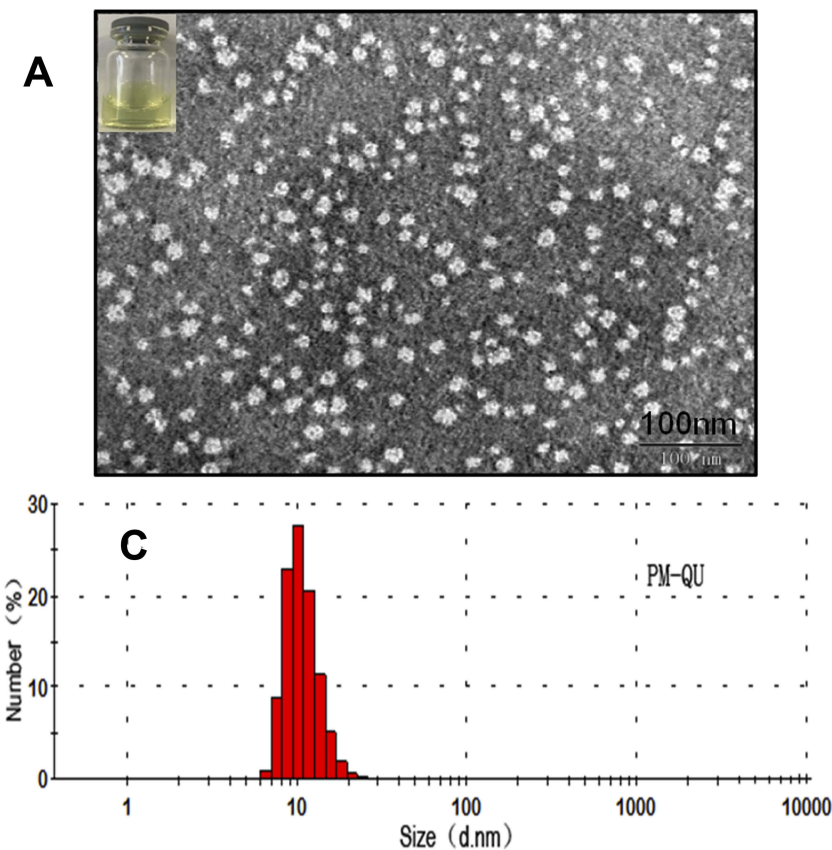

B
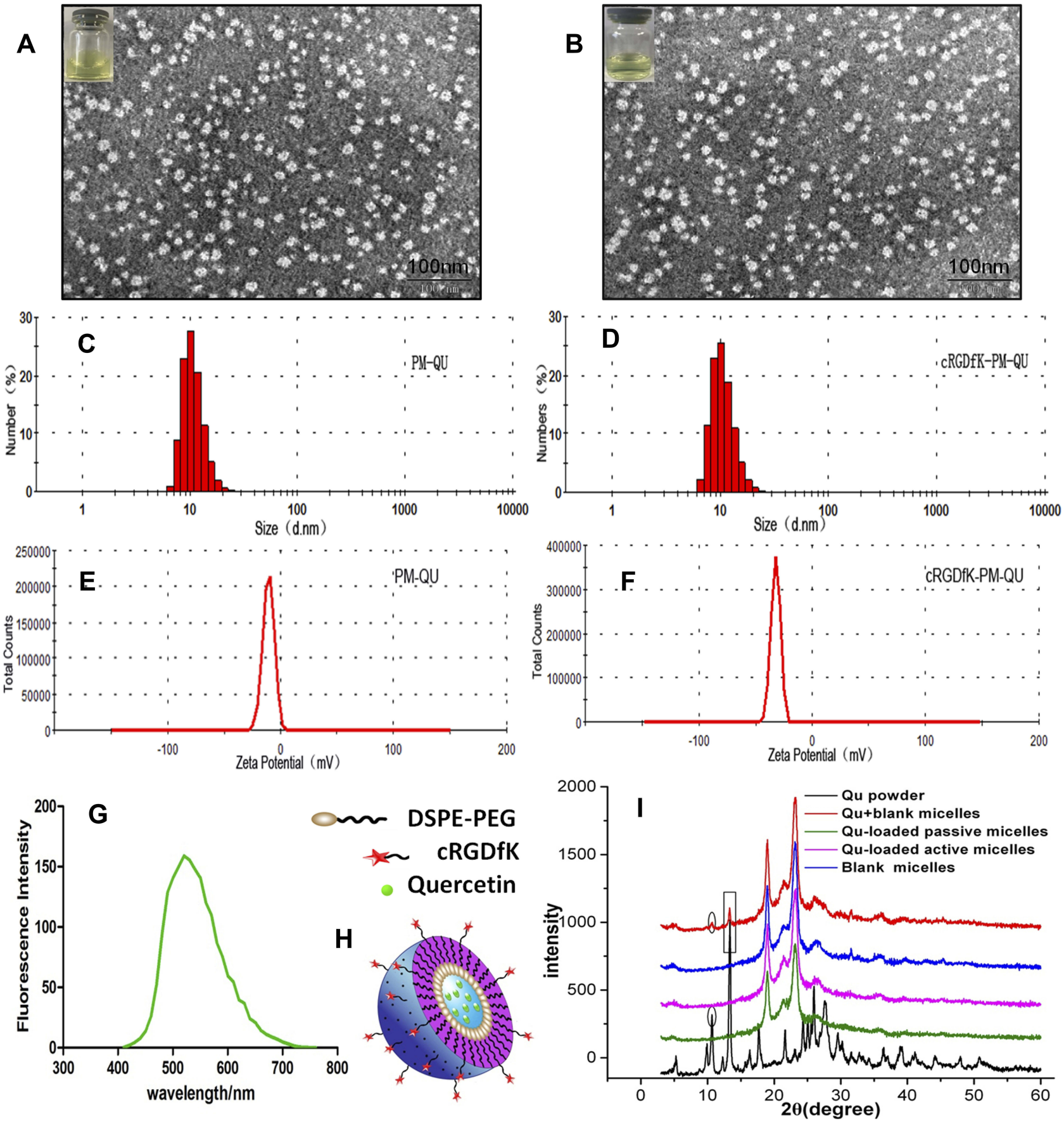

Figure 2 The characteristics of different micelles. (A) Transmission electron microscopy (TEM) image of PM-QU. (B) TEM image of cRGDfK-PM-QU. (C) Particle size distribution of PM-QU surveyed by dynamic light scattering analysis. (D) Particle size distribution of cRGDfK-PM-QU surveyed by dynamic light scattering analysis. (E) Zeta potential of PM-QU. (F) Zeta potential of cRGDfK-PM-QU. (G) The fluorescence spectra of quercetin micelles motivated by $405 \mathrm{~nm}$. (H) Schematic graph of cRGDfK-modified micelles loaded with quercetin (cRGDfK-PM-QU). (I) Powder X-ray diffraction patterns of QU powder, blank micelles of PEG-DSPE, physical mixture of QU with blank micelles, and lyophilized QU-encapsulated micelles, separately. 
that slight negative charges on nanoparticles can contribute to avoiding the non-specific organ uptake and enhancing tumor-specific accumulation. ${ }^{36}$ Figure $2 \mathrm{G}$ shows the fluorescence spectra of quercetin micelles and Figure $2 \mathrm{H}$ depicts the schematic graph of cRGDfK-modified polymeric micelles. Finally, free QU powder, blank PEG-DSPE micelles, the mixture of free QU and blank micelles, and QU-loaded micelles were scanned by the X-ray diffraction (XRD) in Figure 2I. As is shown in Figure 2I, QU power obviously exhibited sharp diffraction peaks at $10.7^{\circ}$ and $13.4^{\circ}$. Similar crystal diffraction peaks of QU were also observed in the physical mixture of QU and blank micelles. Nevertheless, QU-loaded micelles exhibited identical spectra to that of the blank micelles; the diffraction peaks of QU were absent. The phenomenon indicated that QU might exist in amorphous or molecular state in the polymeric micelles.

The physicochemical properties of different micelles are summarized in Table 1. The encapsulation efficiency (EE) of QU in both micelle formulations was over $86 \%$, and its drug concentration could be as high as $1 \mathrm{mg} / \mathrm{mL}$.

\section{Studies On The Interactions Between The Micelles And The $\alpha_{v} \beta_{3}$-Integrin Receptor Overexpressing B 6 Cells}

As a promising technology, SPR can be used to measure the molecular interactions in real time. In this study, SPR was employed to investigate the influence of cRGDfK modification on the interactions between the micelles and the $\alpha_{v} \beta_{3}$-integrin receptor overexpressing B16 cells. ${ }^{37}$ As is demonstrated in Figure 3A, B16 cells were attached on chip surface via covalent binding. Subsequently, with the flow of phosphate buffer containing active or passive micelles, the response signals which indicated the binding ability of the micelles with B16 cells were recorded. For B16 cells, cRGDfK-PM triggered much more strong responses than PM (Figure $3 \mathrm{~B}$ ), indicating that the activetargeting micelles displayed improved binding to the cells. Therefore, via SPR technology, it was observed that cRGDfK modification enhanced the adhesion between the nanocarriers and $\alpha_{v} \beta_{3}$-overexpressing B16 cells.

\section{Uptake Of The Micelles By BI6 Cells}

As shown in Figure $4 \mathrm{~A}$ and B, the B16 cells took up cRGDfK-PM-QU much faster than PM-QU, with the fluorescence intensity in the former group being much stronger than that of the latter group before $800 \mathrm{~s}$. Up to 20 mins, the fluorescence intensities in both groups were comparable. Afterward, further comparison by longer time uptake, the flow cytometry results shown in Figure 4C and D demonstrated that cRGDfK-PM-QU was took up more than PM-QU by B16 cells when the cells were exposed to the micelles for up to $2 \mathrm{hrs}$.

In a word, the earlier results indicated that the cRGDfK modification enhanced the uptake of the micelles by the $\alpha_{\mathrm{v}}$ $\beta_{3}$-integrin receptor overexpressing B16 cells as the peptides were natural ligands of the receptor.

In addition, it was observed that quercetin distributed not only in the cytoplasm but also in the nucleus (Figure S2). The result was in accordance with other reports $^{38}$ that quercetin could specifically bind to DNA and then induce cell apoptosis.
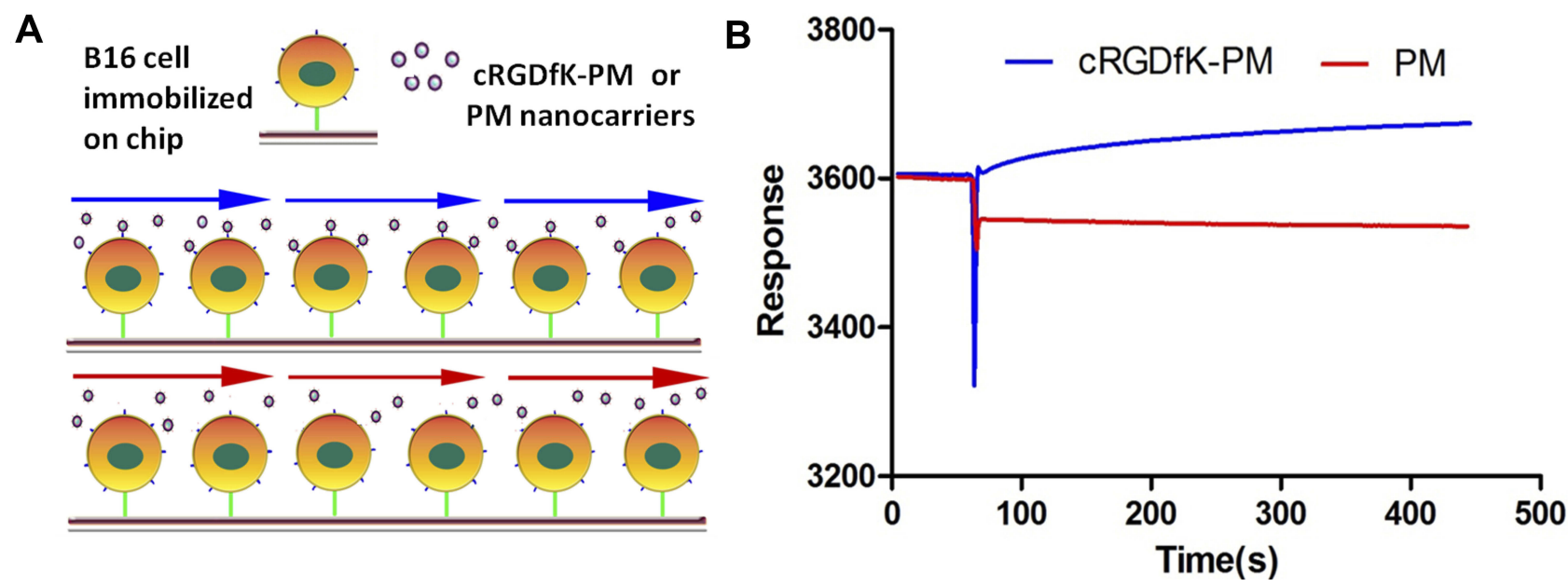

Figure 3 Studies of the micelle-cell interactions by SPR. (A) Schematic graph of a sensor chip bound with BI6 cells in flowing buffer containing the micelles. (B) SPR responses of unmodified micelles and cRGDfK peptides modified micelles with BI6 cells during a 450-s infusion. 


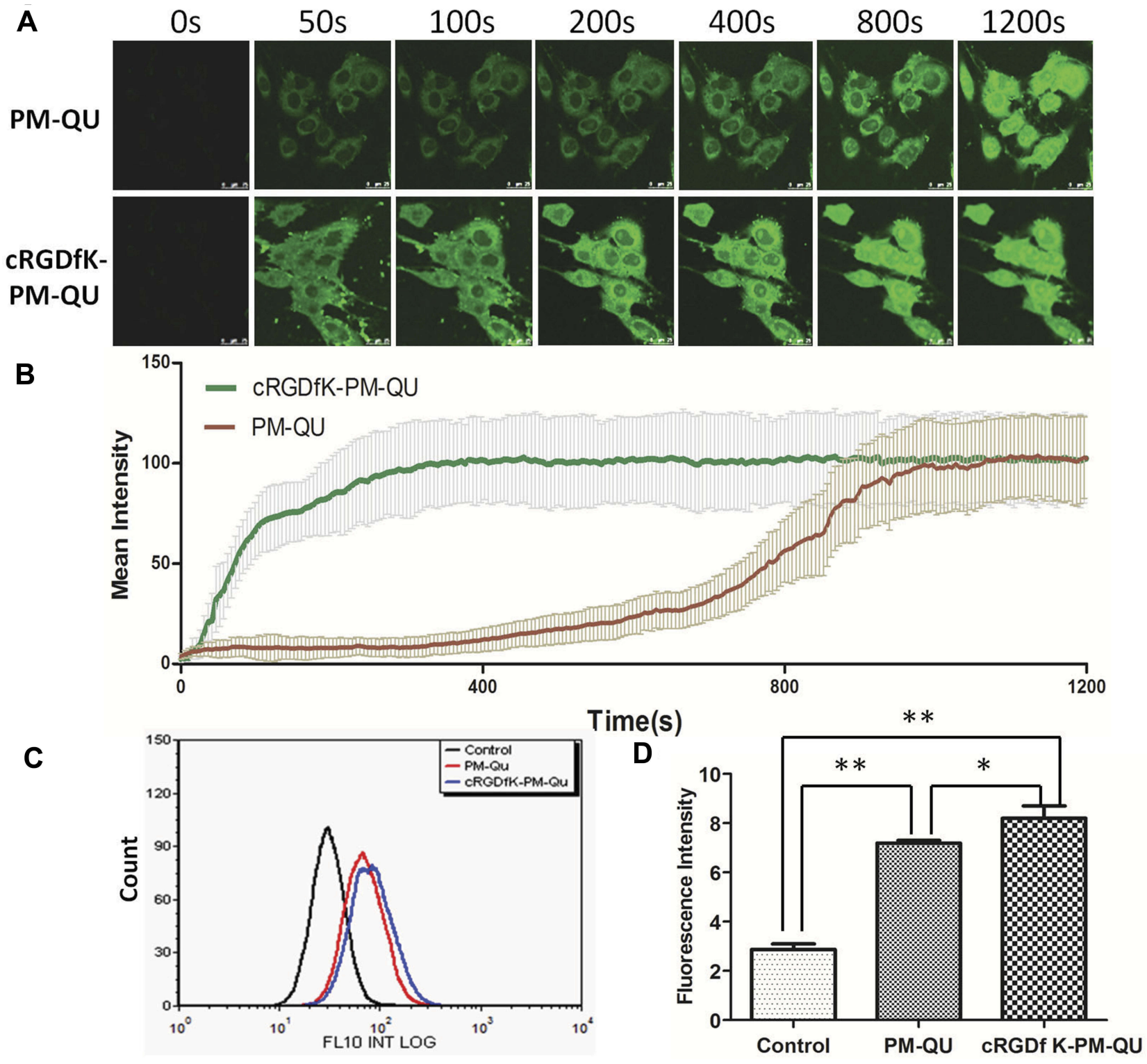

Figure 4 Cellular uptake kinetics of active and passive targeting micelles by BI6 cells. (A) Real-time confocal images of BI6 cells incubated with PM-QU or cRGDfK-PM-QU at $37^{\circ} \mathrm{C}$. The green color indicates quercetin. (B) Plot of confocal quantitative analysis. Each data on the curve indicate the mean fluorescence intensity derived from 12 randomly selected RIOs. (C) Plot of flow cytometry analysis of BI6 cells incubated with PM-QU or cRGDfK-PM-QU at $37^{\circ} \mathrm{C}$ for 2 hrs. (D) Quantitative results of fluorescence intensity. Data are expressed as mean \pm SD $(n=3)$. * means statistically different $(p<0.05)$, ** means highly different $(p<0.0 \mathrm{I})$.

\section{Ex Vivo Antitumor Effects}

$B 16$ cells viability values of QU-loaded micelles are illustrated in Figure 5A. The result showed that B16 cells proliferation could be increasingly suppressed by PM-QU and cRGDfKPM-QU in a dose-dependent manner. Apparently, cRGDfKPM-QU performed much more sensitive cytotoxicity towards B16 cells than PM-QU. Annexin V-FITC/PI double staining test was used to evaluate the apoptosis-inducing effects of the micelles on B16 cells (Figure 5B). After being exposed to PMQU or cRGDfK-PM-QU for $24 \mathrm{hrs,} \mathrm{the} \mathrm{apoptosis} \mathrm{ratio} \mathrm{of} \mathrm{the}$ cells was $22.27 \%$ and $34.98 \%$, respectively. At the same time, PBS-treated cells show a minimal apoptosis ratio of only $7.47 \%$. In accordance with the flow cytometry results, the confocal images also show most positive-stained cells in the cRGDfK-PM-QU group and least positive-stained cells in the control group. The apoptosis ratios between these two micelle-treated cells were statistically different (Figure 5C). To some extent, the greater cell uptake of cRGDfK-PM-QU contributed to the stronger apoptosis-inducing effects on the malignant cells. 

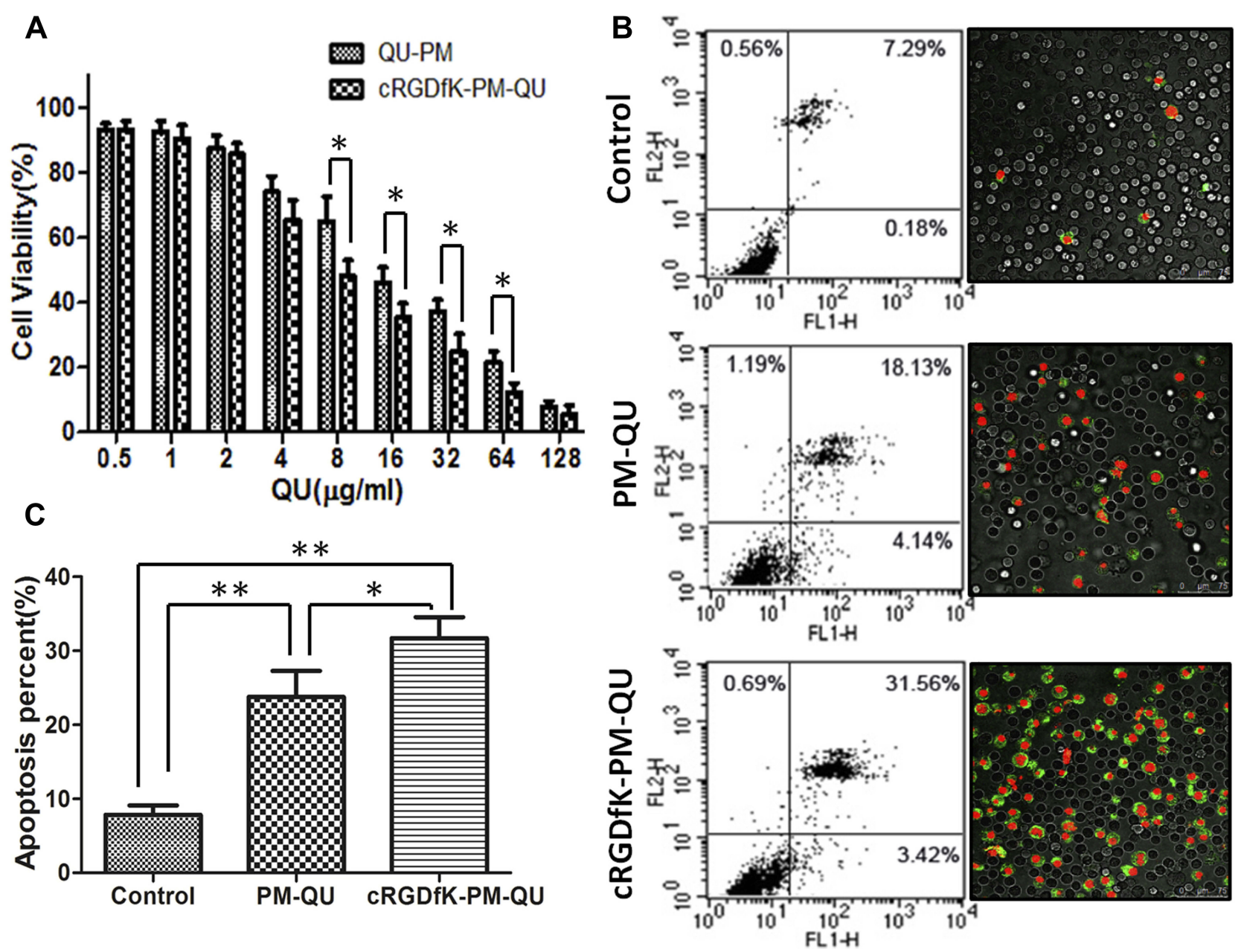

Figure 5 Ex vivo antitumor effects. (A) cytotoxicity analysis after treatment for 24 hrs. (B) Cell apoptosis evaluation by flow cytometry and confocal microscopy. The green fluorescence indicates Annexin $\mathrm{V}$ on the cell membrane and, the red fluorescence indicates $\mathrm{PI}$ in the nucleus. (C) Quantitative analysis of flow cytometry results. Each column represents mean \pm SD $(n=3)$. * means statistically different $(p<0.05)$, ** means highly different $(p<0.01)$.

\section{Distribution In The $\alpha_{v} \beta_{3}$-Overexpressing BI6 Tumor-Bearing Nu/Nu Mice}

In this study, near-infrared fluorescence dye DiR was used to label the micelles to image its distribution in subcutaneous B16 tumor-bearing male nu/nu mice. As shown in Figure 6A, cRGDfK-PM-DiR performed more pronounced fluorescence accumulation in the tumor tissue compared with PM-DiR at different time intervals from $3 \mathrm{hrs}$ to $48 \mathrm{hrs}$. After wholebody optical imaging was finished at $48 \mathrm{hrs}$, the mice were sacrificed, and the tumor tissues and other organs were dissected and imaged immediately (Figure 6B and D). The tumor tissue from the cRGDfK-PM-DiR-treated mice exhibited stronger fluorescence signal than that of the PM-DiRtreated mice. The fluorescence intensity was statistically different (Figure 6C). The above results clearly show that the cRGDfK modification improved the enrichment of the micelles in the malignant tissues due to the well-known active targeting effects. However, the micelles were partially cleared in blood circulation system by reticuloendothelial systems (RES), including liver and spleen (Figure 6E).

\section{Antitumor Effects In Vivo}

In view of enhanced delivery of cRGDfK-PM-QU to $\alpha_{v} \beta_{3^{-}}$ integrin receptor overexpressing B16 tumors in vitro and in vivo, we moved on and conducted the studies on the therapeutic efficiency in nu/nu mice bearing B16 melanoma. As shown in Figure 7A, during the intravenous administration period up to 10 days, compared to the saline group, both the QU groups suppressed the tumor growth, especially, cRGDfK-PM-QU which had stronger inhibition effects on tumor growth than PM-QU. The results could be explained by the improved accumulation of the micelles in the tumors after cRGDfK modification in the previous survey. Finally, the tumor mass from different groups was weighed to validate that 

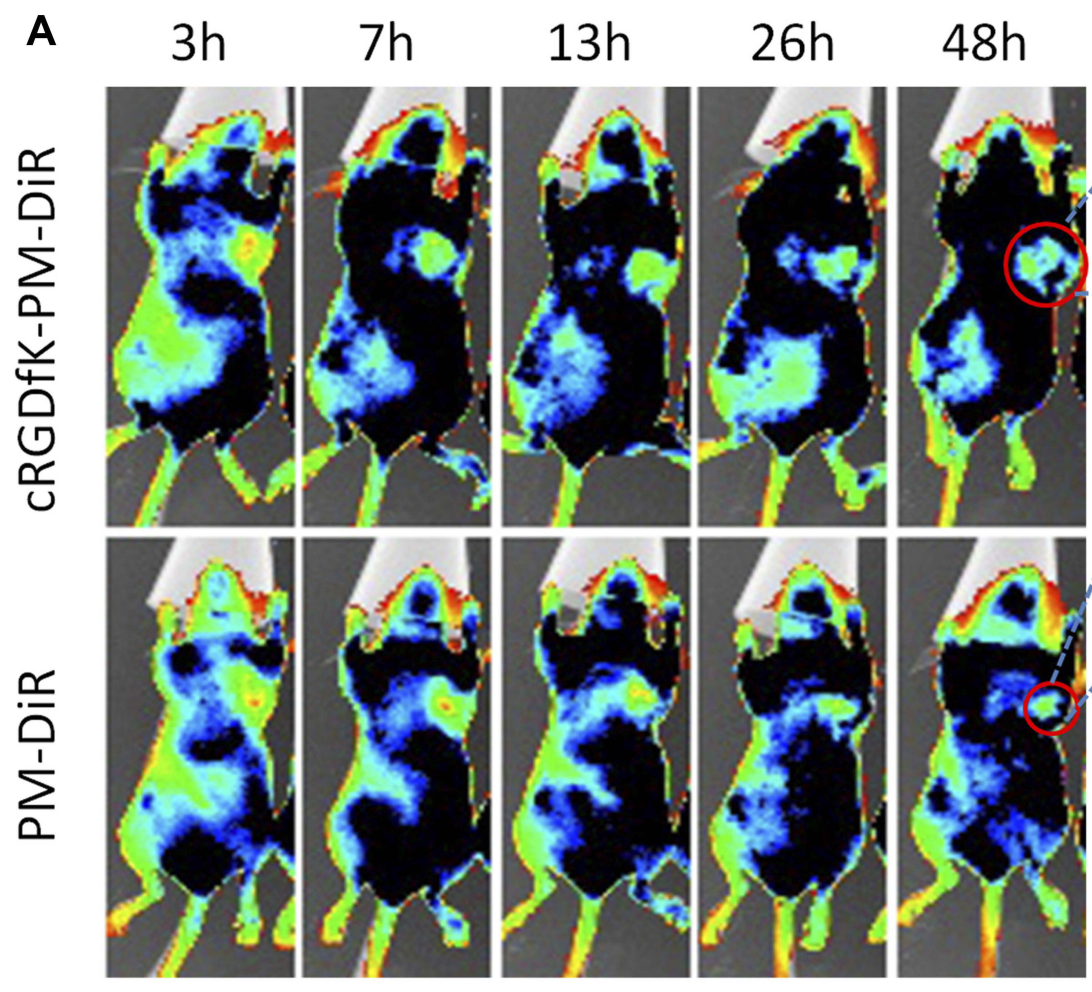

B
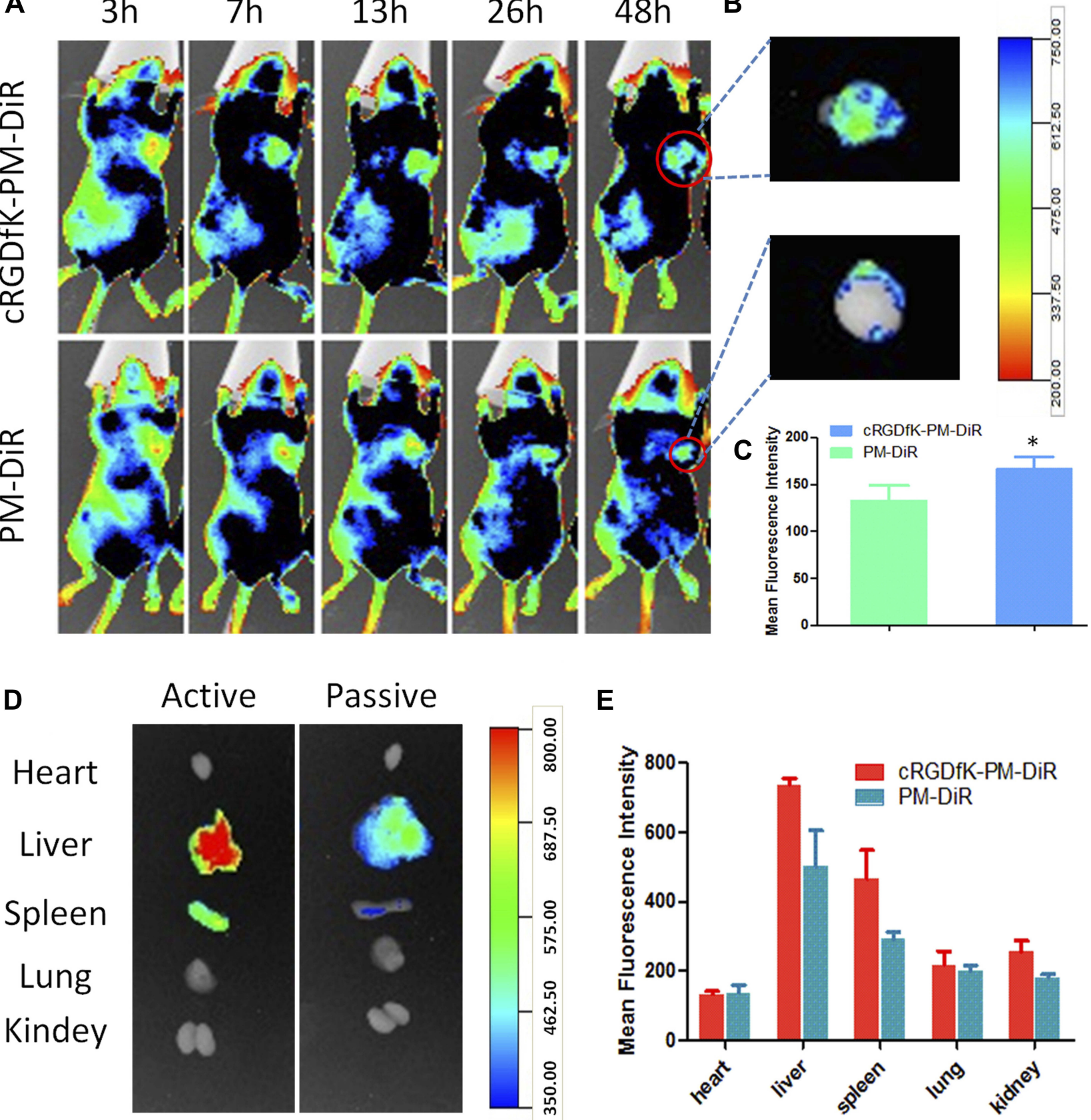

E

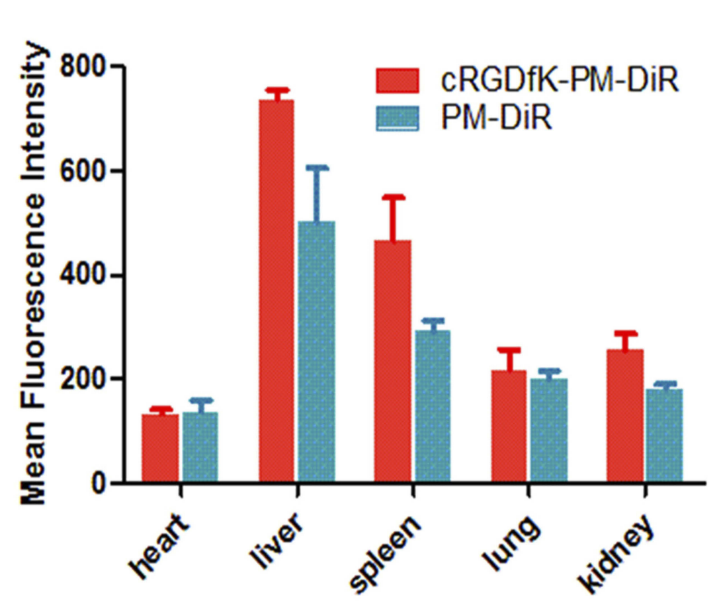

Figure 6 Distribution of the micelles in BI6-tumor-bearing male nu/nu mice (A) Near-infrared fluorescence images of the tumor-bearing mice after being i.v. treated by cRGD-PM-DiR or PM-DiR. Red circles designate the mice tumor region. (B) Near-infrared fluorescence images of the collected tumor tissues at 48 hrs after being i.v. treated by cRGD-PM-DiR or PM-DiR. (C) The semi-quantitative analysis of tumor fluorescence signal at 48 hrs after administration of cRGDfK-PM-DiR or PM-DiR. Data represent mean $\pm S D(n=3)$ and * indicates statistic difference $(p<0.05)$. (D) Near-infrared fluorescence images of the main organs excised from $B \mid 6$ tumor-bearing mice 48 hrs after the micelles treatment. (E) The semi-quantitative analysis of fluorescence signal in the organs excised from BI6 tumor-bearing mice 48 hrs after administration of cRGDfK-PM-DiR or PM-DiR. Data represent mean \pm SD $(n=3)$.

cRGDfK-PM-QU demonstrated superior antitumor activity than PM-QU in B16 tumor-bearing mouse model (Figure 7B).

\section{In Vivo Toxicity Studies}

Finally, the safety of both QU formulations was evaluated in B16 tumor-bearing mice. Figure 8A shows that obvious weight loss was absent during the whole treatment period. The H\&E staining of the main organs confirmed that both formulations did not induce obvious systemic toxicity (Figure 8B). In detail, PM-QU caused slight pulmonary damage, characterized as enlarged alveoli, cell reduction on the alveolar wall, and a narrowed pulmonary alveoli 
A

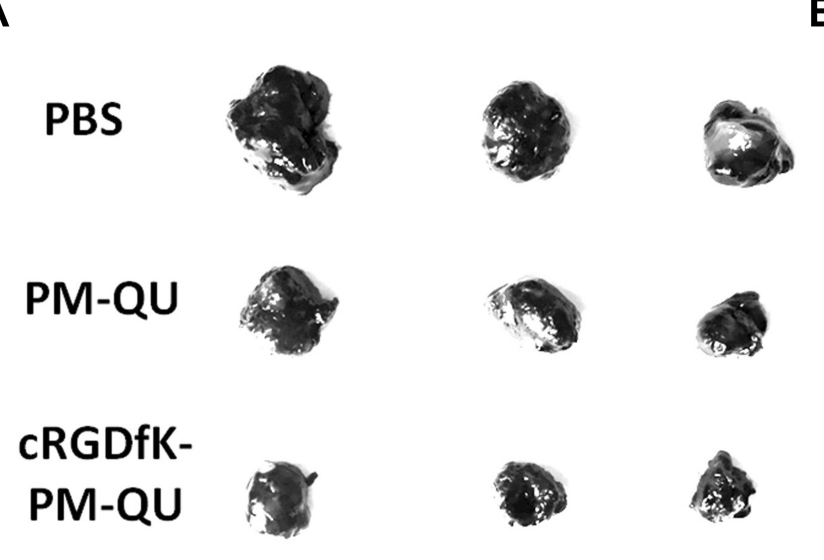

B

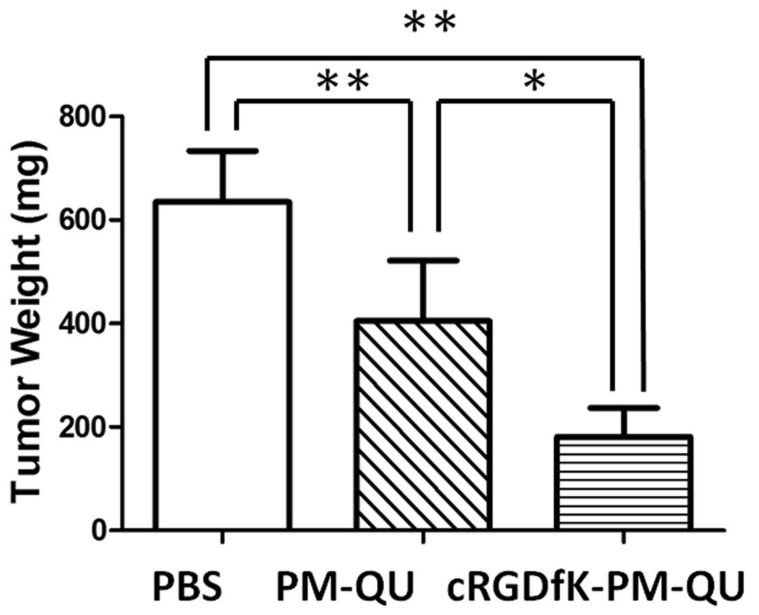

Figure 7 In vivo antitumor effects. (A) The photos of the excised BI6 melanoma mass. (B) The weights of the BI6 melanoma masses. Each column represents mean \pm SD $(n=3)$ * indicates statistic difference $(p<0.05)$, ** indicates highly different $(p<0.01)$.

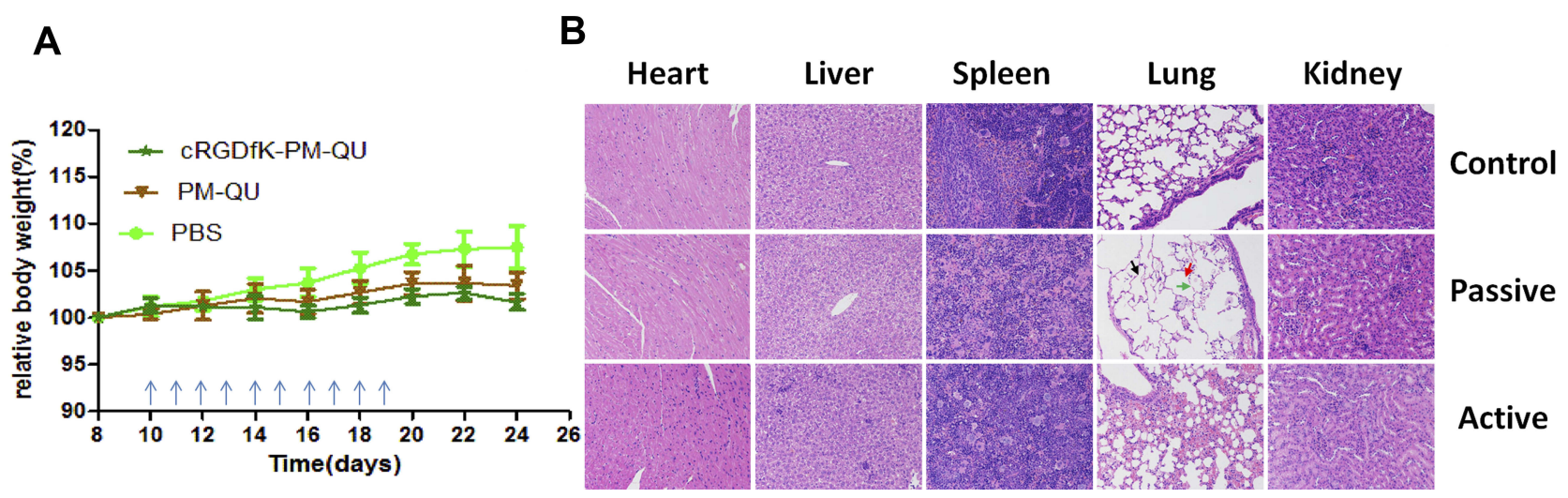

Figure 8 Toxicity studies of the micelles in BI6 tumor-bearing mice. (A) Body weight profiles of the mice. Arrows point out the injection time. (B) H\&E staining of the main organs.

compartment. Meanwhile, cRGDfK-PM-QU did not induce detectable damage to the lung tissue, indicating the superior compatibility of cRGDfK-PM-QU.

\section{Conclusion}

Quercetin as a natural product has triggered increasing interest in the antitumor field. In our study, quercetin was successfully encapsulated inside the cRGDfK-modified micelles by thin-film hydration method and its aqueous solubility increased up to $1.0 \mathrm{mg} / \mathrm{mL}$. In vitro study suggested a uniform small-size particle for PM-QU and cRGDfK-PM-QU. The surface cRGDfK modification had little influence on physicochemical property of the micelles. SPR assay revealed that cRGDfK-modified micelles could bind to B16 cells via cRGDfK- $\alpha_{\mathrm{v}} \beta_{3}$ integrin receptor interactions, which induced higher intracellular uptake by B16 cells and enhanced apoptosis. We also confirmed that cRGDfK modification could improve the enrichment of the micelles in the malignant tissues, thus resulting in better therapeutic effects on B16 tumorbearing mice and less systemic toxicity compared with PM-QU. In particular, carrier materials of quercetinloaded micelles are approved by FDA, making our formulation have high clinical potential. Further studies are needed to investigate the dosing strategy in order to yield optimal therapeutic efficacy of cRGDfK-PM-QU micelles.

\section{Acknowledgments}

This research was supported by the National Natural Science Foundation of China (No. 81760760, No. 81690264), the National Basic Research Program of China (2015CB932 100), Inner Mongolia Autonomous Region Natural Science 
Foundation (No. 2016MS0847), Scientific Research Planning Project of Health and Family Planning Commission of Inner Mongolia Autonomous Region (No. 201701048), the Science and Technology Planning Project of Inner Mongolia Autonomous Region (No. 201502101).

\section{Author Contributions}

All authors contributed to data analysis, drafting or revising the article, gave final approval of the version to be published, and agree to be accountable for all aspects of the work.

\section{Disclosure}

The authors report no conflicts of interest in this work.

\section{References}

1. Russo GL, Russo M, Spagnuolo C, et al. Quercetin: a pleiotropic kinase inhibitor against cancer. Cancer Treat Res. 2014;159:185-205.

2. Tan WF, Lin LP, Li MH, et al. Quercetin, a dietary-derived flavonoid, possesses antiangiogenic potential. Eur J Pharmacol. 2003;459:255-262. doi:10.1016/S0014-2999(02)02848-0

3. Niu G, Yin S, Xie S, et al. Quercetin induces apoptosis by activating caspase-3 and regulating Bcl-2 and cyclooxygenase-2 pathways in human HL-60 cells. Acta Biochim Biophys Sin (Shanghai). 2011;43:30-37. doi:10.1093/abbs/gmq107

4. Yuan Z, Long C, Junming T, Qihuan L, Youshun Z, Chan Z. Quercetin-induced apoptosis of HL-60 cells by reducing PI3K/Akt Mol Biol Rep. 2012;39:7785-7793. doi:10.1007/s11033-012-1621-0

5. Sakao K, Fujii M, Hou DX. Acetyl derivate of quercetin increases the sensitivity of human leukemia cells toward apoptosis. Biofactors. 2009;35:399-405. doi:10.1002/biof.53

6. Yu CS, Lai KC, Yang JS, et al. Quercetin inhibited murine leukemia WEHI-3 cells in vivo and promoted immune response. Phytother Res. 2010;24:163-168. doi:10.1002/ptr.2841

7. Li N, Sun C, Zhou B, et al. Low concentration of quercetin antagonizes the cytotoxic effects of anti-neoplastic drugs in ovarian cancer. PLoS One. 2014;9(7):e100314. doi:10.1371/journal.pone.0100314

8. Yang W, Muneeb Ahmed MD, Tasawwar B, et al. Radiofrequency ablation combined with liposomal quercetin to increase tumour destruction by modulation of heat shock protein production in a small animal model. Int $J$ Hyperthermia. 2011;27(6):527-538. doi:10.3109/02656736.2011.582474

9. Zhi-pingYuan L-JC, Fan L-Y, Tang M-H, Yang G-L, Zhao X, Wei Y-Q. Liposomal quercetin efficiently suppresses growth of solid tumors in murine models. Clin Cancer Res. 2006;12(10):3193-3199. doi:10.1158/ 1078-0432.CCR-05-2365

10. Hashemzaei M, Far AD, Yari A, et al. Anticancer and apoptosis-inducing effects of quercetin in vitro and in vivo. Oncol Rep. 2017;38(2):819-828. doi:10.3892/or.2017.5766

11. Hu K, Miao L, Goodwin TJ, Li J, Liu Q, Huang L. Quercetin remodels the tumor microenvironment to improve the permeation, retention, and antitumor effects of nanoparticles. ACS Nano. 2017;11 (5):4916-4925. doi:10.1021/acsnano.7b01522

12. Bhattaram VA, Graefe U, Kohlert C, Veit M, Derendorf $\mathrm{H}$. Pharmacokinetics pharmacokinetics and bioavailability of herbal medicinal products. Phytomedicine. 2002;9(Suppl 3):1-33. doi:10.1078/14 33-187X-00210

13. Dewhirst MW, Secomb TW. Transport of drugs from blood vessels to tumour tissue. Nat Rev Cancer. 2017;17(12):738-750. doi:10.1038/ nrc. 2017.93
14. Banerjeea A, Qi J, Gogoi R, Wong J, Mitragotri S. Role of nanoparticle size, shape and surface chemistry in oral drug delivery. $J$ Control Release. 2016;238:176-185. doi:10.1016/j.jconrel.2016.07.051

15. Hua S, BPharm E, Marks JJ, Schneider B, Keely S. Advances in oral nano-delivery systems for colon targeted drug delivery in inflammatory bowel disease: selective targeting to diseased versus healthy tissue. Nanomedicine. 2015;11(5):1117-1132. doi:10.1016/j.nano.2015.02.018

16. Tan B-J, Liu Y, Chang K-L, Bennie KW, Lim G, Chiu NC. Perorally active nanomicellar formulation of quercetin in the treatment of lung cancer. Int J Nanomedicine. 2012;7:651-661. doi:10.2147/IJN.S26538

17. Polireddy K, Dong R, Reed G, et al. High dose parenteral ascorbate inhibited pancreatic cancer growth and metastasis: mechanisms and a phase I/IIa study. Sci Rep. 2017;7(1):17188. doi:10.1038/s41598017-17568-8

18. Pridgen EM, Alexis F, Omid C. Farokhzad Polymeric nanoparticle drug delivery technologies for oral delivery applications. Expert Opin Drug Deliv. 2015;12(9):1459-1473. doi:10.1517/17425247.2015.1018175

19. Verrax J, Calderon PB. Pharmacologic concentrations of ascorbate are achieved by parenteral administration and exhibit antitumoral effects. Free Radical Biol Med. 2009;47(1):32-40. doi:10.1016/j. freeradbiomed.2009.02.016

20. Zhang L, Chan JM, Gu FX, et al. Self-assembled lipid-polymer hybrid nanoparticles: a robust drug delivery platform. ACS Nano. 2008;2(8):1696-1702. doi:10.1021/nn800275r

21. Che J, Okeke CI, Hu Z-B, Jing X. DSPE-PEG: a distinctive component in drug delivery system. Curr Pharm Des. 2015;21 (12):1598-1605. doi:10.2174/1381612821666150115144003

22. Yu C, He B, Xiong M-H, et al. The effect of hydrophilic and hydrophobic structure of amphiphilic polymeric micelles on their transport in epithelial MDCK cells. Biomaterials. 2013;34(26):6284-6298. doi:10.1016/j.biomaterials.2013.05.006

23. Jee J-P, McCoy A, Mecozzi S. Encapsulation and release of amphotericin $\mathrm{B}$ from an $\mathrm{ABC}$ triblock fluorous copolymer. Pharm Res. 2012;29(1):69-82. doi:10.1007/s11095-011-0511-9

24. Geng S, Yang B, Wang G, Qin G, Wada S, Wang J-Y. Two cholesterol derivative based PEGylated liposomes as drug delivery system, study on pharmacokinetics and drug delivery to retina. Nanotechnology. 2014;25(27):275103. doi:10.1088/0957-4484/25/27/275103

25. Zhao B, Wang X-Q, Wang X-Y, et al. Nanotoxicity comparison of four amphiphilic polymeric micelles with similar hydrophilic or hydrophobic structure. Part Fibre Toxicol. 2013;10:47. doi:10.1186/ 1743-8977-10-47

26. Zhang L, Gu FX, Chan JM, Wang AZ, Langer RS, Farokhzad. OC. Nanoparticles in medicine: therapeutic applications and developments. Clin Pharmacol Ther. 2008;83(5):761-769. doi:10.1038/sj.clpt.61 00400

27. Bogdanowich-Knipp SJ, Chakrabarti S, Siahaan TJ, Williams TD, Dillman RK. Solution stability of linear vs. cyclic RGD peptides. J Pept Res. 1999;53(5):530-541. doi:10.1034/j.1399-3011.1999.000 52.x

28. Ogawa M, Hatano K, Oishi S, et al. Direct electrophilic radiofluorination of a cyclic RGD peptide for in vivo alpha(v)beta3 integrin related tumor imaging. Nucl Med Biol. 2003;30(1):1-9. doi:10.1016/ S0969-8051(02)00387-6

29. Haubner R, Wester H-J, Reuning U, et al. Radiolabeled alpha(v)beta3 integrin antagonists: a new class of tracers for tumor targeting. $J$ Nucl Med. 1999;40(6):1061-1071.

30. Aumailley M, Gurrath M, Müller G, Calvete J, Timpl R, Kessler H. Arg-Gly-Asp constrained within cyclic pentapeptides: strong and selective inhibitors of cell adhesion to vitronectin and laminin fragment P1. FEBS Lett. 1991;291(1):50-54. doi:10.1016/0014-5793(91) 81101-D

31. Gurrath M, Müller G, Kessler H, Aumailley M, Timpl R. Conformationactivity studies of rationally designed potent anti-adhesive RGD peptides. Eur J Biochem. 1992;210(3):911-921. doi:10.1111/j.14 32-1033.1992.tb17495.x 
32. Pfaff M, Tangemann K, Muller B, et al. Selective recognition of cyclic RGD peptides of NMR defined conformation by alpha IIbbeta 3, alpha v beta 3 and alpha 5 beta 1 integrins. J Biol Chem. 1994;269(32):20233-20238.

33. van der Pluijm G, Hans JMV, Ivanov B, et al. Bone sialoprotein peptides are potent inhibitors of breast cancer cell adhesion to bone. Cancer Res. 1996;56(8):1948-1955.

34. Delforge D, Gillon B, Art M, Dewelle J, Raes M, Remacle J. Design of a synthetic adhesion protein by grafting RGD tailed cyclic peptides on bovine serum albumin. Lett Pept Sci. 1998;5:87-91. doi:10.1007/ BF02443445

35. Cai D, Gao W, Bing H, et al. Hydrophobic penetrating peptide PFVYLI-modified stealth liposomes for doxorubicin delivery in breast cancer therapy. Biomaterials. 2014;35(7):2283-2294. doi:10.1016/j. biomaterials.2013.11.088
36. He C, Hu Y, Yin L, Tang C, Chunhua Y. Effects of particle size and surface charge on cellular uptake and biodistribution of polymeric nanoparticles. Biomaterials. 2010;31(13):3657-3666. doi:10.1016/j. biomaterials.2010.01.065

37. Peiris D, Markiv A, Curley GP, Dwek MV. A novel approach to determining the affinity of protein-carbohydrate interactions employing adherent cancer cells grown on a biosensor surface. Biosens Bioelectron. 2012;35(1):160-166. doi:10.1016/j.bios.2012.02.037

38. Mitrasinovic PM, Palakshan PT, Tripathi S, Tripathi AN. On the affinity and specificity of quercetin for DNA. Med Chem. 2013;9 (2):193-202. doi:10.2174/1573406411309020004

\section{Publish your work in this journal}

The International Journal of Nanomedicine is an international, peerreviewed journal focusing on the application of nanotechnology in diagnostics, therapeutics, and drug delivery systems throughout the biomedical field. This journal is indexed on PubMed Central, MedLine, CAS, SciSearch ${ }^{\mathbb{R}}$, Current Contents ${ }^{\mathbb{R}} /$ Clinical Medicine, $^{2}$
Journal Citation Reports/Science Edition, EMBase, Scopus and the Elsevier Bibliographic databases. The manuscript management system is completely online and includes a very quick and fair peer-review system, which is all easy to use. Visit http://www.dovepress.com/ testimonials.php to read real quotes from published authors. 